Universidad Nacional de La Plata.

Facultad de Humanidades y Ciencias de la Educación.

Centro de Investigaciones Socio Históricas

\title{
El hospital como espacio de trabajo. Algunas claves para el análisis del conflicto sindical en salud pública
}

\author{
The hospital as a workspace. Some keys to analyze the trade \\ dispute in public health
}

\begin{abstract}
Anabel Angélica Beliera *
* Instituto de Investigaciones en Humanidades y Ciencias Sociales (IdIHCS)

CONICET - Universidad Nacional de La Plata (UNLP), Argentina | anabeliera@gmail.com
\end{abstract}

\section{PALABRAS CLAVE}

Empleo público

Hospital

Acción colectiva

Tramas políticas

Sindicalismo

\section{KEYWORDS}

Public employment

Hospital

Collective action

Political networks

Trade unionism

\section{RESUMEN}

En este artículo se analiza la trama política del conflicto sindical en salud pública, a partir del estudio de la experiencia de los trabajadores del Hospital Provincial Neuquén entre los años 2005 y 2013. A partir de una estrategia metodológica cualitativa, se realizó una investigación situada con un abordaje relacional donde se plasmó el entramado de relaciones en el que se desenvuelve el conflicto laboral. Aquí se analiza la articulación entre conflictos laborales y la disputa más general por la gestión de las políticas públicas, las regulaciones especiales que legislan el trabajo hospitalario y la presencia de la "comunidad" a quienes está orientado este servicio. Estas reflexiones llevan a dialogar con los estudios sindicales en la post-convertibilidad, realizando un aporte relativo al estudio del conflicto de trabajadores estatales y de una provincia del interior del país. Se argumenta a favor de una perspectiva relacional que entienda los conflictos sindicales y huelgas en el marco de las relaciones locales que integran los trabajadores. 


\section{Introducción}

A partir del año 2003 se ha vivido en Argentina una reactivación del conflicto laboral liderado por los trabajadores del sector formal y con un renovado protagonismo de las organizaciones sindicales tradicionales. Se trató de un momento ofensivo, en donde las demandas de las organizaciones sindicales no sólo buscaron retener los beneficios del pasado defendiéndose contra los despidos, desempleo y flexibilización, sino que buscaron activamente aumentos salariales, cobertura contractual, sindicalización de los trabajadores y distribución de la ganancia (Antón, Cresto, Rebón, \& Salgado, 2010; Etchemendy \& Collier, 2008).

Los elementos que explican la reactivación del conflicto sindical son múltiples. Algunas investigaciones resaltaron el impacto de la modificación de la estructura económica a partir del crecimiento económico y la recuperación de la tasa de ganancia en una cadena de producción con alta demanda e intenso ritmo de trabajo. Esto promovió el resurgimiento de las huelgas como mecanismos para disputar los aumentos de salario y mejorías en las condiciones laborales (Etchemendy \& Collier, 2008). Sin embargo, si bien es cierto que la transformación de la sociedad salarial en nuestro país es un factor que favorece la constitución del conflicto social se centre en los procesos laborales, no es un elemento suficiente para explicar la emergencia de acciones colectivas en este ámbito.

Un segundo conjunto de investigaciones dentro de la bibliografía centró la atención en las características propias de los actores que se movilizaban, como las particularidades de las organizaciones políticas, los recursos organizativos que tenían a disposición, la productividad política de ciertas identidades y/o los posicionamientos políticos de los diferentes actores implicados en el conflicto. En relación a los posicionamientos políticos de los distintos grupos intervinientes en el conflicto laboral, algunas investigaciones centraron su atención en el análisis de las cúpulas (sindicales, empresariales y estatales) mientras otras prefirieron orientarse al estudio de las organizaciones sindicales de base situadas en los espacios de trabajo. Por un lado, las investigaciones que analizaron las dinámicas de las centrales sindicales y las negociaciones colectivas de trabajo a nivel de cúpulas resaltado que la proliferación de negociaciones colectivas se debió tanto al accionar de las organizaciones sindicales como a la participación estatal en la regulación de la relación capital-trabajo (Anigstein, 2012; Etchemendy \& Collier, 2008; Palomino \& Trajtemberg, 2007). El aumento de los convenios colectivos negociados a nivel de las cúpulas consolidó un sistema de fijación colectiva del salario y de las condiciones de uso de la fuerza de trabajo (Campos \& Campos, 2011; Coscia, 2011; Etchemendy, 2011; Gómez, 2006; González \& Haidar, 2009). 1 Por otro lado, en este periodo se registra una gran proliferación de conflictos protagonizados por las bases sindicales, en donde se destacan las tensiones presentes entre las comisiones internas y la estructura sindical centralizada característica del sindicalismo argentino (Santella, 2011; Scolnik, 2009). Dentro de los estudios centrados en el sindicalismo de base, fueron mayoritarios los casos analizados en provincia de Buenos Aires: el Cuerpo de Delegados del subterráneo de Buenos Aires (Arias \& Haidar, 2008; Compañez, Ventrici, \& Voscos, 2012; Lenguita, 2011; Varela, 2016), la Junta Interna del Hospital Garrahan (Duhalde, 2011; Scolnik, 2009), y la Comisión interna de Kraft-Terrabusi (Cambiasso, 2013; Varela, 2016).

A pesar de que ha habido una creciente preocupación por analizar la dinámica del conflicto gremial a partir del 2003, aún es necesario profundizar en algunos aspectos que han quedado sub-representados en los análisis de las ciencias sociales.

En primer lugar, son escasas las investigaciones sobre el conflicto sindical en las provincias del interior del país pues la mayoría de los investigadores dirigieron su mirada hacia la provincia de Buenos Aires o a la escala nacional (analizando las dinámicas de las centrales y federaciones, los convenios colectivos de trabajo, los vínculos con el Estado Nacional). Considero que el análisis de experiencias locales que no han sido estudiadas en profundidad por las ciencias sociales puede aportar una gran riqueza al debate académico. 
En ese sentido, algunas particularidades que presenta el caso de Neuquén son interesantes para el análisis de las acciones colectivas de los trabajadores. Allí se desarrollaron experiencias que tuvieron gran impacto en la política nacional: las ‘puebladas' de Cutral-Có y Plaza Huincul luego de la privatización de la empresa petrolera estatal Yacimientos Petrolíferos Fiscales (YPF) constituyen uno de los orígenes del movimiento piquetero; la ocupación y puesta en producción de la fábrica Zanón luego de su declaración de quiebra, el movimiento de trabajadores desocupados, los paros docentes del sindicato ATEN. $\underline{2}$ La complejidad de estos procesos requiere una mirada atenta a la configuración histórica de demandas orientadas al Estado y la constitución de subjetividades políticas de resistencia, en la que se combinaron procesos de larga data con otros más coyunturales. Estos procesos se articularon a partir de demandas claramente orientadas al Estado provincial, en donde diversos sectores de la población neuquina disputaron a los miembros del gobierno provincial las formas de gestión de lo publico en esta provincial. En estas contiendas, los trabajadores estatales han tenido una participación muy activa, y particularmente los trabajadores de salud pública han sido protagónicos.

Un segundo aspecto que ha quedado sub-representado en los estudios sobre el conflicto sindical en este periodo es el análisis de los espacios de trabajo estatales, pues en general los estudios se centraron en los sindicatos del sector privado. El sindicalismo en los ámbitos estatales de trabajo adquiere particularidades que resultan interesantes para la conceptualización del conflicto gremial. $\underline{3}$ Primeramente, en estos casos el conflicto sindical no se trata de un enfrentamiento lineal entre trabajadores y patrones, pues el Estado en tanto empleador es una entidad abstracta que no se de forma directa a una persona o grupo de personas definidos. En tanto entidad abstracta, no sólo se presenta formalmente como un empleador sino también como un garante de los derechos de la población. En segundo lugar, la distinción entre patrones y trabajadores no está dada por la posesión o desposesión de los medios de producción, sino que se fundamenta en procesos políticos y en dinámicas de identificación y diferenciación entre distintos grupos de personas. Es decir, que unos sean identificados como empleadores y otros como empleados no es una cuestión que se desprenda únicamente de la situación contractual de compra y venta de la utilización de la fuerza de trabajo sino que se fundamenta también en procesos de construcción cultural y simbólica. En tercer lugar, el trabajo estatal es la única rama de actividad que admite más de un sindicato representativo con personería jurídica (que en la mayoría de los casos son ATE y UPCN). A diferencia de los espacios laborales dentro de empresas o fabricas privadas, en los ámbitos estatales de trabajo encontramos competencia entre dos o más organizaciones sindicales reconocidas legalmente por el Estado como interlocutores representativos de los trabajadores del sector. Finalmente, en la organización sindical de empleados públicos se articulan tanto demandas corporativas como demandas sobre el modo de gestión de las políticas estatales. Por ende, el conflicto no sólo afecta a empleadores y empleados sino también a diversas personas y sectores de la población al menos en su carácter de ciudadanos o contribuyentes (Diana Menéndez, 2007). El análisis de estos espacios de trabajo puede representar un aporte al estudio de las acciones sindicales y al análisis de la reactivación del conflicto laboral en el periodo de la post convertibilidad.

El objetivo de este artículo es entonces presentar algunas claves para conceptualizar el conflicto sindical en salud pública, a partir del análisis de la experiencia de los trabajadores del Hospital Provincial Neuquén Dr. Castro Rendón (HPN) entre los años 2005 y 2013. Para ello se diseñó una estrategia metodológica cualitativa con perspectiva etnográfica, basada en la realización de observaciones sistemáticas y participantes en el espacio hospitalario, que fueron registradas en notas organizadas y estructuradas. Asimismo, se realizaron 37 entrevistas semi-estructuradas en profundidad a 29 trabajadores del hospital (algunos de ellos fueron entrevistados más de una vez). El trabajo de campo se llevó a cabo durante los años 2010, 2012 y 2013.

Hemos decidido centrar el análisis en el HPN por la importancia que ha tenido este hospital en la política sanitaria de la provincia y en la experiencia sindical de los trabajadores del sistema de salud: es único 
hospital de referencia provincial, lo que implica que es prestador público de todo el territorio (y no de una región particular de la provincia); es la institución de máxima complejidad del sistema -nivel VIII- por lo que dispone de la totalidad de las especialidades médicas, además de mantener actividades permanentes de docencia e investigación; es un hospital-escuela en donde terminan su especialización numerosos residentes de la carrera de medicina. En lo relativo al conflicto sindical, se trata del hospital que más trabajadores emplea en la provincia de Neuquén; es el único hospital que cuenta con comisiones internas de diversos sindicatos en el lugar de trabajo; $\stackrel{4}{\text { y }}$ por su ubicación en el centro de la ciudad capital de la provincia, se tornó epicentro de actos políticos, concentraciones, movilizaciones, festivales de denuncia, y asambleas de trabajadores.

Este artículo se divide en tres apartados. En el primer apartado analizaremos el hecho de que el sistema de salud pública tenga una regulación especial para el ejercicio de derecho a huelga, por tratarse de un servicio esencial. En el segundo apartado, se analiza cómo es referenciada la comunidad de pacientes en las prácticas y discursos de los trabajadores, marcando disputas con los funcionarios del gobierno provincial. En el tercer apartado revisaremos la imbricación entre los aspectos institucionales y disruptivos que tienen lugar en un ámbito estatal de trabajo. El análisis de estos elementos nos permitirá argumentar a favor de una perspectiva de análisis del conflicto sindical que no se centre únicamente en el estudio de los momentos de huelga, sino que atenga a la dinámica cotidiana de relaciones en las que se inserta la participación sindical de los trabajadores. Para ello se retoman algunas investigaciones locales que privilegian la mirada sobre los procesos y tramas de politicidad, suspendiendo la pregunta por las formas de acción y la constitución de actores políticos (Fernández Álvarez, 2007; Manzano, 2008). Se procura tener un enfoque analítico "que descentre la mirada de la organización de la acción para iluminar las dinámicas del campo político y social dentro del cual la movilización ocurre” (Manzano, 2008, p. 5), lo que supone un abordaje relacional que atienda a la configuración y redefinición de escenarios de la disputa.

\section{El conflicto sindical en un servicio esencial.}

Una parte importante de las investigaciones sindicales en el periodo de la post-convertibilidad se ha orientado a cuantificar la magnitud de la reactivación del conflicto gremial a partir del año 2003 a través de la medición de diversos indicadores como la cantidad de huelgas desarrolladas, la evolución de las afiliaciones sindicales, la cantidad de convenios colectivos de trabajo homologados. $\frac{5}{}$ Si bien existen dificultades en estas mediciones, $\underline{6}$ la cuantificación de estos indicadores ha sido central para estudiar la renovada centralidad del conflicto laboral a escala nacional y el importante rol que jugaron en ese proceso las organizaciones sindicales tradicionales. Sin embargo, consideramos que estos estudios cuantitativos deben ser complementados con análisis cualitativos que analicen de forma relacional y situada los conflictos protagonizados por los trabajadores. Aquí argumentaremos que las huelgas y medidas de fuerza llevadas a cabo por los sindicatos deben ser entendidas en el marco de relaciones de las que forman parte. Para ello, analizaremos el desarrollo de medidas de fuerzas en el HPN y las dificultades implicadas en la realización de huelgas con retención de servicios en este sector.

La modificación de las políticas laborales y las relativas a la salud pública son temas de discusión tanto en las asambleas sindicales como en los espacios de trabajo, locales gremiales y movilizaciones de los trabajadores del HPN. La sensación de pérdida de derechos individuales y sociales, la percepción de la capacidad de agencia para defenderlos y la diferenciación que establecen con "los funcionarios del gobierno" son elementos que convergen en el proceso de agudización del conflicto en el hospital y en la decisión de iniciar un "plan de lucha".

Sin embargo, muchas veces las medidas de fuerza comienzan con la realización de "paros con fichado" en los que los trabajadores concurren al lugar de trabajo pero suspenden algunas actividades, como la atención 
en los consultorios externos, los estudios de alta complejidad y las cirugías programadas. En general, a estos paros se le suman acciones complementarias para difundir el conflicto, como caminatas y volanteadas por los barrios, establecimiento de carpas-hospitales en la calle para realizar prácticas ambulatorias de rutina, movilizaciones, radios abiertas, abrazos simbólicos al hospital. Obviamente, este tipo de paros ("con fichado") no tiene la misma eficacia política que una huelga general clásica con suspensión total de las actividades laborales, pero la decisión de realizar este tipo de medidas se debe a un conjunto de condicionamientos presentes en este entramado particular. Es decir, no se debe a una evaluación estratégica de la eficacia de esta medida de fuerza ni a los cálculos racionales que establecen los actores sobre los costos y beneficios para implicarse en la acción, sino del conjunto de compromisos y relaciones que están implicados en el trabajo cotidiano.

¿Por qué los trabajadores utilizan los "paros con fichado" en lugar de realizar una huelga con retención de servicios? Algunos elementos a tener en cuenta son las regulaciones formales que tiene el ejercicio de derecho a huelga de los trabajadores de salud pública, las prácticas organizativas de los trabajadores de este hospital y los sentimientos que atraviesan los trabajadores.

En consonancia con la normativa internacional, el Estado argentino establece que la atención sanitaria es un servicio esencial y determina para estos casos la obligación de garantizar la prestación de servicios mínimos que eviten su plena interrupción (artículo 24 de ley 25.877, sancionada en el año 2004). Se consideran esenciales los servicios sanitarios y hospitalarios, la producción y distribución de agua potable, energía eléctrica y gas, y el control del tráfico aéreo. La importancia de estos servicios radica en que la interrupción de su actividad puede poner en peligro la vida, la seguridad o la salud de toda o parte de la población. Se trata de servicios públicos de importancia trascendental, conforme los criterios de los organismos de control de la Organización Internacional del Trabajo (OIT).

Dado que la interrupción total del servicio es imposible, existe una regulación especial para el ejercicio de derecho a huelga de los trabajadores de la salud pública: Por un lado, las huelgas nunca pueden ser totales puesto que un conjunto de trabajadores debe mantenerse en el espacio de trabajo para garantizar el funcionamiento del sector. Es decir, los trabajadores de salud pública no pueden ejercer plenamente el derecho a huelga por tener la obligación de mantener "guardias mínimas" en los centros de salud y hospitales. Esto no es necesariamente un problema en todas las huelgas, pues no siempre participa el total de los trabajadores; pero está siempre presente como una preocupación al momento de decidir dar comienzo a un plan de lucha. Por otro lado, el Estado mantiene la potestad del Ministerio de Trabajo de fijar unilateralmente la extensión de los servicios mínimos ante la falta de acuerdo entre las partes y se le otorga además la facultad de incrementarlos cuando, a su juicio, resultasen insuficientes. ${ }^{7}$

José, dirigente de la Junta Interna de la Asociación de Trabajadores Estatales -ATE- en el HPN, señala como una de las principales dificultades dentro de la dinámica sindical del sector el hecho de que el gobierno los pueda acusar de no atender a la población durante las huelgas:

En salud hay que tener mucho equilibrio para la pelea. Porque si esto fuera una fábrica, la cosa es mucho más fácil: si vos producís, cerras la fábrica, y el patrón tiene que cambiar la forma, su diálogo. [En salud] hay que tener mucho equilibrio para no caer a lo que el gobierno nos quiere llevar, que es culparlos a los trabajadores del deterioro del sistema y del cierre de los hospitales. Entonces hay que tener mucho equilibrio en lo público. (José, auxiliar técnico del sector de electro medicina, miembro de la ATE)

José explica que las medidas de fuerza tienen que ser “equilibradas” para que no se acuse a los trabajadores de "querer cerrar los hospitales". Esto vuelve inteligible el hecho que muchas veces realicen medidas de fuerza en las que de todas formas asisten al lugar de trabajo, atienden a los pacientes y sólo reducen algunas 
de sus tareas (como la atención por consultorios externos).

La decisión de realizar "paros con fichado" en los que no se interrumpe plenamente la actividad laboral es una consecuencia de estas particularidades pues con esta modalidad de protesta, la atención de los pacientes no se suspende completamente. De esta forma, los trabajadores dirigen sus demandas al gobierno provincial sin posibilitar a que éste actúe únicamente como garante de la atención sanitaria y colocándolo en la obligación de dar respuesta al reclamo laboral. Es decir, los funcionarios del gobierno provincial no pueden presentarse únicamente como los encargados de mantener los servicios sanitarios para los ciudadanos (cuestión que sucedería si los trabajadores iniciasen una huelga con retención de servicios) sino que son interpelados en tanto empleadores de los trabajadores hospitalarios. Al no suspenderse la atención de pacientes durante este tipo de medidas de fuerza, se los interpela exclusivamente como destinatarios del reclamo laboral.

Sin embargo, muchas veces las medidas de fuerza se extienden por falta de convocatoria a una mesa conciliatoria o porque, una vez convocada, no es posible arribar a un acuerdo. Cuando los "paros con fichado" se extienden durante varios días (o meses) sin tener un impacto político significativo, los trabajadores evalúan la posibilidad de comenzar a realizar "paros sin fichado". Éstos implican mayores trastornos ya que la atención de pacientes queda limitada a las urgencias ingresadas por las guardias e internaciones. Los planteles de trabajadores se ven reducidos en todos los sectores del hospital, dependiendo del nivel de adhesión a la medida de fuerza. En los momentos de mayor adhesión, se tienen que organizar guardias mínimas en cada uno de los sectores para garantizar la atención indispensable de los pacientes internados y de las guardias de emergencias.

Esto conlleva problemas organizativos puesto que se deben organizar planteles de trabajadores que no realicen huelga y puedan garantizar el mantenimiento de los servicios mínimos. A estas dificultades organizativas se les suman las dificultades afectivas que atraviesan los trabajadores, ya que son situaciones que implican un sentimiento de angustia debido a que se puede afectar a los pacientes que asisten al hospital. Los trabajadores se enfrentan con grandes conflictos éticos y políticos debido a la vulnerabilidad de la población que concurre al HPN, ya que quienes reciben atención en los hospitales públicos son generalmente los sectores más empobrecidos de la población, sufriendo necesidades básicas insatisfechas y siendo desprotegidos por otras instituciones estatales antes de llegar a este sanatorio. Los trabajadores relatan el abatimiento que supuso dejar las labores debido a las consecuencias que podía tener para "la comunidad" la realización de huelgas en el sector. Esta cuestión es expresada como uno de los principales obstáculos para llevar adelante acciones de este tipo:

Acá hay seres humanos, hay personas... y acá puede estar tu tía, tu mamá, tu hermano. Y yo como delegado, al frente de la asamblea te tengo que dar una respuesta, como ser humano. Y si yo tengo un conflicto, vos no tenés la culpa. (José, auxiliar técnico de electro-medicina, miembro de ATE)

Es muy difícil. Dejar los sectores [de trabajo] es muy difícil. (...) Lo que se hace es, por ejemplo, siempre que hay emergencias, se entra y se sale. Entonces, por ejemplo, en el 2005 hubo un chiquito que tuvo un accidente, y corría riesgo su vida. Entonces se vino, se entró a trabajar para cubrir a ese paciente que corría riesgo su vida, hasta que se lo derivó, hasta que se consiguió cama para derivarlo. (...) Entonces, cuesta salir a pelear, porque cuesta dejar los pacientes. Pero hay que salir, es así. Y eso se vive como una cuestión difícil. (María, administrativa, miembro de ATE)

El hecho de que no haya mayor cantidad de huelgas con retención de servicios en el HPN no se debe entonces a la ausencia de conflictos o debates políticos dentro del hospital, sino al conjunto de obligaciones y 
responsabilidades que tienen los trabajadores en relación a los pacientes.

Las reflexiones sobre las particularidades del trabajo hospitalario en relación al conflicto gremial llaman la atención sobre un aspecto importante de la dinámica sindical: las huelgas y medidas de fuerza desarrolladas por los trabajadores deben ser analizadas en articulación con el entramado de relaciones en las que se insertan; es decir, analizando la trama que ocupan los trabajadores y que modifican partir de su intervención. En este caso, es fundamental considerar que se trata de un servicio esencial cuya regulación legal impone condicionamientos al desarrollo de medidas de fuerza con retención de servicios, razón por la cual los trabajadores inician sus acciones de protesta con medidas como los "paros con fichados". La ausencia de paros con retención de actividades no implica que no haya conflicto sindical o político dentro del hospital, sino que es necesario registrar esa conflictividad laboral en las redes cotidianas del trabajo hospitalario.

Obviamente que es central analizar las transformaciones económicas y sociales estructurales, el tipo de demandas que reclaman los trabajadores y/o los procesos de construcción de sí mismos como sujetos políticos que intervienen en el espacio público. Pero el análisis de la conflictividad sindical a partir de las huelgas o protestas en el espacio público debe ser complementado con el estudio de la conflictividad laboral en los propios espacios de trabajo. De esta forma, la huelga en tanto medida de fuerza puede ser analizada desde una perspectiva situada y relacional.

En suma, si asumimos una perspectiva relacional se torna evidente que las formas que asume la acción no depende únicamente de las estrategias de los trabajadores sino que debe ser entendida en relación los condicionamientos que regulan su entramado político. Para comprender las prácticas políticas de los trabajadores del HPN no basta con evaluar únicamente sus estrategias políticas y acciones de protesta en el espacio público sino que es necesario tener analizar las regulaciones legales que las condicionan.

\section{Entramados: los trabajadores, la comunidad y los funcionarios.}

De la misma forma que es importante analizar los condicionamientos legales del ejercicio del derecho a huelga, en este caso se torna fundamental considerar a los otros actores y grupos de personas que conforman la trama de relaciones del hospital. La vida política dentro del hospital es un proceso siempre abierto y conflictivo, en el que intervienen múltiples grupos.

La teoría de Norbert Elías resulta útil para resaltar esta dimensión relacional y procesual de la experiencia. Elias (1999) afirma que los actos de las partes -individuos o grupos- deben ser entendidos como un proceso de entramado en el que los individuos dependen de otros de forma recíproca. El autor apela a la metáfora del juego como una herramienta para analizar la dimensión relacional que condiciona las decisiones de los individuos: las decisiones no se producen nunca de forma exclusivamente personal dado que están limitadas por las jugadas del resto. El concepto de figuración hace referencia entonces a la totalidad "integrada por los jugadores interdependientes y el juego que juegan entre sí” (Elias, 1999, p. 99); es decir, el modelo cambiante que constituyen los jugadores como totalidad. La interdependencia entre las distintas posiciones genera procesos regulares que se imponen a cada jugador como procesos impersonales y que no dependen de la voluntad de los individuos (Criado, 2008). El concepto de figuración permite entonces dar cuenta de las cadenas de interdependencia que vinculan grupos de Nosotros y Ellos en relación al juego de disputa de poder espacio-temporal siempre conflictivo e inestable.

El conflicto político del que participan los trabajadores del HPN se debe analizar en relación a los otros grupos que conforman el entramado, entendiéndolos como posiciones relacionales dentro de una misma estructura de juego. Las demandas de los trabajadores no pueden evaluarse como un fin que dependa únicamente de su decisión individual o sectorial, sino que debe ser analizada en relación al desenvolvimiento cotidiano del proceso de trabajo y a las relaciones que se establecen con dos grupos de personas distintos: 
“la comunidad neuquina” y "los funcionarios del gobierno".

Veamos primero a que se refieren los trabajadores cuando afirman que tienen mucho vinculo "con la comunidad”. Es usual que las organizaciones de trabajadores del HPN tengan un vínculo muy fluido con otras organizaciones del arco político neuquino, con quienes realizan actividades en forma conjunta. En diversas asambleas del sector usualmente se hacen presentes representantes de otras organizaciones políticas e incluso participan como oradores para manifestar su apoyo o proponer alguna actividad. Estas organizaciones no se limitan a los sindicatos del sector (como puede ser la conducción provincial ATE, el Sindicato de Profesionales de la Salud Publica Neuquina -SiProSaPuNe-, Sindicato de Enfermeros -SEN-, o representantes de organizaciones federativas como la CTA), sino que también incluye a organizaciones de derechos humanos, sindicatos de otras ramas de actividad, personalidades destacadas de la política local, Madres de Plaza de Mayo filial Alto Valle, miembros del movimiento estudiantil secundario y universitario, integrantes del Movimiento Ecuménico por los Derechos Humanos, el equipo de Pastoral Social del obispado de Neuquén, y representantes provinciales de partidos de la oposición al gobierno.

Respecto a esta articulación, hay algunos eventos que marcaron la experiencia sindical de los trabajadores de salud pública y son relatadas aun hoy como momentos significativos de su articulación política con otras organizaciones. Por ejemplo, la huelga desarrollada en el año 2005 fue un punto de inflexión en la organización sindical del sector tanto por su extensión e intensidad como por la extensa red de alianzas que forjaron con otros sectores de la población neuquina. $\underline{8}$ Esas relaciones se plasmaron incluso en marcas materiales en el hospital: En uno de los pasillos del hospital se encuentra pegado un libro mural realizado en cerámicos con poemas de Juan Gelman, que fue realizado por los obreros de la empresa recuperada Fabrica Sin Patrones (ex Zanón) y donado a los trabajadores en huelga como un gesto de solidaridad. También de ese año los trabajadores recuerdan y relatan vívidamente algunos momentos en que personas importantes de la política extra-regional se hicieron presentes en el HPN para manifestar su apoyo, como aquellas ocasiones en las que el premio Nobel de la Paz Adolfo Pérez Esquivel y Vicente Zito Lema se presentaron en asambleas para dirigir unas palabras a los trabajadores y manifestar su apoyo.

El vínculo con otras organizaciones del entramado político neuquino se materializó en más de una oportunidad en la conformación de mesas multisectoriales, como se ve en los siguientes relatos:

Una multisectorial hacíamos del hospital, la convocamos nosotros donde venían todos los partidos de distintas organizaciones, todos los otros sindicatos, y el sindicato ceramista, y los ocupados y los desocupados, y hasta las comisiones vecinales venían. (Oscar, enfermero, miembro de ATE)

En un conflicto vino Pérez Esquivel. Fue un momento muy emocionante. [Vino] invitado por la Asociación de Derechos Humanos. Porque nosotros también tenemos muchas migas con la gente de derechos humanos, con las Madres de Plaza de Mayo... estamos con toda la gente... acá se hicieron mucho más allá que reuniones gremiales... logramos la multisectorial, que era una organización política porque vos ahí nucleás a la sociedad. Dentro de todas estas asambleas que veníamos garroteados, fue muy emocionante tener al Premio Nobel de la Paz. Si, habló... ¡viste Pérez Esquivel cómo habla! La verdad que le llegó a todo el mundo. (Daniel, carpintero del sector de mantenimiento, miembro de ATE)

En este último fragmento se remarca que en el hospital se realizaron "mucho más que reuniones gremiales” y se pone como ejemplo el hecho de haber participado en una multisectorial en la que se nucleaba " $a$ la sociedad". Estos vínculos políticos con otras organizaciones no se basan únicamente en una estrategia de alianza basada en un cálculo racional de los trabajadores del HPN, sino que activa sentimientos: los trabajadores del HPN afirman que se “emocionan” y que "les llegan” las palabras de los oradores en las 
asambleas (en este caso refiriéndose a Pérez Esquivel).

Un párrafo especial merece la organización Familiares, Amigos y Vecinos de Enfermos Agrupados (FAVEA) que está integrada por los allegados de pacientes que conformaban la lista de espera para recibir atención quirúrgica en el HPN. Como consecuencia de la crisis sanitaria a la que llegó la provincia de Neuquén a finales del año 2004 por la falta de anestesiólogos, se conformó en la provincia una cuantiosa lista de espera de pacientes que esperaban su turno para poder operarse en el hospital. Estos pacientes movilizaron sus redes externas al hospital (en sus barrios, grupos de amigos, familia, etc.) y conformaron una organización FAVEA-. Los trabajadores del HPN señalan la importancia de esta organización en la ampliación de sus redes de movilización:

A raíz de la crisis de la anestesia, se forma acá un listado como de pacientes para operarse, una lista de casi mil doscientos pacientes esperando. Y se conforma una organización que se llama FAVEA, de familiares y amigos para la salud pública, en defensa de la salud. Y con ella sí, articulamos un montón de cosas... marchas, conflictos, mesas, con ellos tenemos mucho acercamiento. (Ana, médica pediatra, miembro de SiProSaPuNe)

Le ponen FAVEA porque tenían familiares y amigos que les suspendían la cirugía, que estaban un mes, dos meses, un año esperando que se los opere, y de esa manera se forma esta asociación digamos. Y que hoy sigue estando, sigue firme. Hay gente que sigue trabajando en FAVEA, sigue haciendo sus reclamos, ellos siempre estuvieron con nosotros. (...) En algún momento el gobierno los convocó como para demostrarles de que les quiso abrir las puertas como para que ellos trabajaran, y en realidad lo que ellos veían es que nunca había respuesta... o sea, había reuniones tras reuniones tras reuniones, pero en realidad las propuestas nunca llegaban, ¿viste? (Federico, enfermero, miembro de ATE)

Los trabajadores del HPN presentan públicamente sus demandas y promueven actividades de denuncia en forma conjunta con otras organizaciones del entramado político neuquino. En los fragmentos de entrevistas de esta medica clínica y este enfermero se presenta el vínculo de los trabajadores del HPN con la organización FAVEA como una relación “de apoyo” y armonía, en oposición a la actuación del gobierno que "los convoco" pero "nunca les daba respuesta" a sus reclamos.

El hecho de que diversas organizaciones políticas y sociales participen activamente de los conflictos sindicales de trabajadores estatales ha sido señalada también por Diana Menéndez (2007), quien destaca que en estos conflictos hay una suerte de 'competencia' de toda la población en la 'cosa pública', una especie de intervención política de la comunidad, como mínimo desde su carácter de contribuyente. En salud publica esto adquiere una particularidad más: dado que la salud pública es un servicio esencial que no puede ser suspendido por las consecuencias que puede tener sobre la vida de la población, cuando las huelgas se masifican, la relación con "la comunidad" se vuelve decisiva.

Sin embargo, aquí quisiéramos resaltar una cuestión más: esta apelación a “la comunidad” no se mantiene siempre en el plano de la generalidad, sino que se establecen disputas por delimitar sus fronteras (quienes quedan incluidos y excluidos del colectivo político al que se apela). Esta articulación es una construcción variable y depende de cómo se enfrenten los distintos grupos en la lucha política. A través de la apelación a "la comunidad", los trabajadores intentan delimitar fronteras entre distintos espacios y tiempos del Estado, creando identidades sociales a partir de las cuales se definen a sí mismos y se diferencian de otros (uniendo simbólicamente los “trabajadores” a la “comunidad”, y diferenciándose de los “funcionarios de gobierno”).

Como todas las articulaciones discursivas que hablan sobre la totalidad (la comunidad, la sociedad) esta disputa política hace uso del mecanismo de exclusión discursiva. Laclau y Mouffe (1985) afirman que la lucha política se da principalmente en el terreno discursivo, entendiendo al discurso como la totalidad 
estructurada resultante de la práctica articulatoria que establece una relación entre elementos. Generalmente la idea de discurso está asociada al signo comunicado por vía escrita u oral, pero en la concepción de estos autores el discurso es una relación social que incluye elementos lingüísticos y extralingüísticos. El discurso es un complejo de elementos en el que cual las relaciones juegan un rol constitutivo (Laclau, 2004, 2005). El discurso es un intento de imponer un orden al mundo -orden del que naturalmente carece-, pues recorta la infinitud de lo posible para imprimir estructuras a las relaciones sociales. Este proceso es contingente, pues la construcción del orden resulta siempre modificada por la variación de las fuerzas sociales intervinientes.

Laclau y Mouffe (1985) afirman que la articulación discursiva de "la sociedad" es un intento de producir una objetividad que se reproduzca sin momentos de ruptura o antagonismo. La sociedad como totalidad constituye un objeto que es a la vez imposible y necesario (Laclau, 2005): es necesario porque sin algún tipo de cierre, por más precario que fuera, no habría ninguna significación ni identidad; pero es imposible porque el cierre total nunca llega a realizarse dado que cualquier proceso social implica conflictos y antagonismos.

Estas reflexiones son útiles para volver inteligible la presencia de la comunidad en el entramado político de los trabajadores del HPN. Dado que esta articulación no tiene fundamentos esenciales sino contingentes, es importante analizar los sentidos que implican para grupos histórica y localmente situados. Cuando dicen "comunidad" lo hacen teniendo presente las tomas de posición del Otro, y luchan por establecer qué elementos quedan excluidos. Este mecanismo se grafica a continuación:

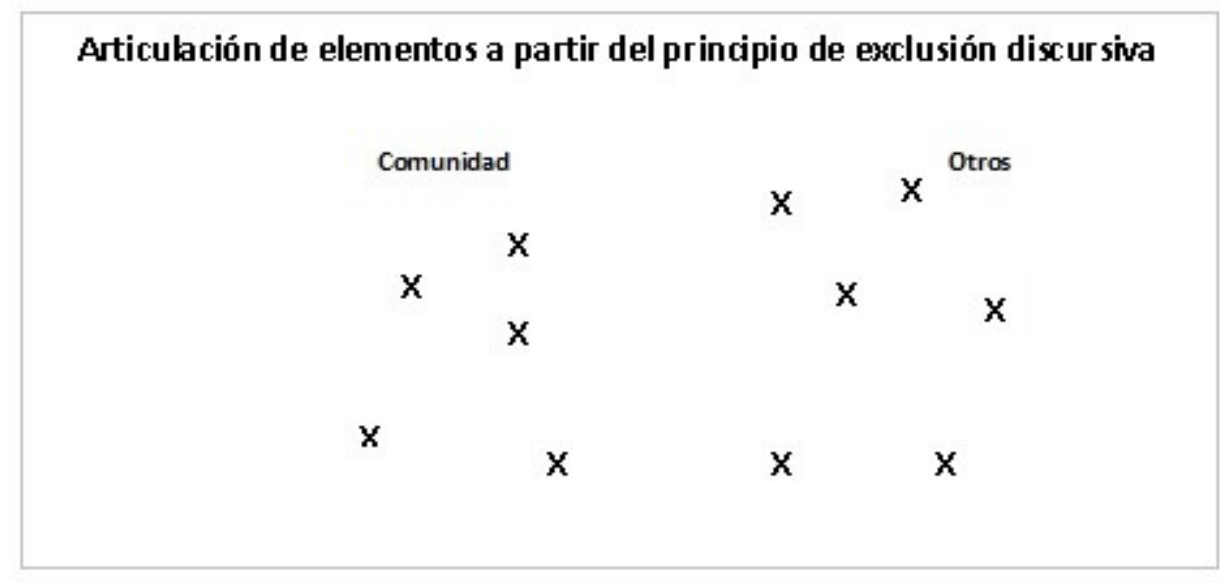

El círculo del grafico no está completamente cerrado para mostrar dos cuestiones: en primer lugar, que esta articulación no responde a ninguna cualidad esencial o natural de los elementos, sino que es producto de un mecanismo de diferenciación social; en segundo lugar, que es una articulación que puede variar y agrupar otros elementos. No es un cierre total del grupo, sino una cuestión transitoria plausible de ser modificada.

La apelación a "la comunidad" que realizan los trabajadores debe ser analizada en relación a la disputa que establecen con los funcionarios de gobierno. Por un lado, los funcionarios del gobierno presentan las huelgas de los trabajadores del HPN como un enfrentamiento entre los sindicatos y la comunidad neuquina. Es usual que el poder ejecutivo intente controlar las huelgas vigilando que se garanticen los servicios mínimos de atención. Han sido varias las ocasiones en que el gobierno provincial realizó denuncias judiciales a los trabajadores en las que se los acusaba de no garantizar los planteles mínimos de atención en los hospitales públicos y/o efectuó sumarios administrativos por abandono de personas a los huelguistas. A estas medidas formales se sumaron medidas informales, como declaraciones a la prensa acusando a los trabajadores de abandonar a los enfermos en los hospitales y/o de pedir aumentos de sueldos desmedidos. Aquí los trabajadores del HPN aparecían como un actor que dañaba a la comunidad desde fuera, y la exclusión simbólica de los sindicatos del interior de la comunidad neuquina pretendía generar la unificación de un 
Nosotros-neuquinos-victimas del accionar sindical. Al enfrentar a los trabajadores y la comunidad, se buscaba romper la fuerza equivalencial (Laclau y Mouffe, 1985) de las demandas de los trabajadores del HPN con las de otros sectores de la sociedad neuquina como otros sindicatos de trabajadores estatales y organizaciones políticas del arco militante neuquino.

En oposición a los funcionarios de gobierno, los trabajadores del HPN presentaban a "la comunidad" como articulada a "los trabajadores". En el siguiente fragmento de la publicación "Esceptiscemia”, revista sindical realizada por el Sindicato de Profesionales de la Salud Publica Neuquina -SiProSaPuNe-, se afirma que los funcionarios ocultan el deterioro de la salud pública y le "mienten a la comunidad" con el objetivo de "enfrentarlos a los trabajadores":

Entonces, los funcionarios que salen a declarar que 'tenemos una Salud Pública excelente', ocultan las interminables listas quirúrgicas (...). Y, fundamentalmente, salen a mentirle a la comunidad, pretendiendo hacer pasar a los trabajadores en conflicto como aprovechadores de la bonanza neuquina, haciendo publicar sueldos que ninguno de nosotros llega a cobrar; y defendiendo en cambio la obsecuencia mostrada por funcionarios políticos adeptos que, pese a la manifiesta ineptitud con que manejan la gestión pública, se las ingenian para llenar su patrimonio con propiedades. (Escepticemia, número 67/ 2005)

En este fragmento se refiere a los "funcionarios" como "políticos adeptos". La diferenciación que los trabajadores establecen respecto a los funcionarios no refiere únicamente a la jerarquía de los puestos sino a las distintas posiciones políticas que implican. Esto permite entender que la distinción entre funcionarios y trabajadores refiere fundamentalmente a la trama política del espacio hospitalario y no meramente a una división de tareas entre distintos puestos laborales dentro del Estado. Es decir, no se establece únicamente sobre aspectos contractuales del trabajo sino que implica un proceso deconstrucción social y cultural.

De esta forma, para contrarrestar la idea de que las huelgas del sector pueden implicar un daño para "la comunidad", los trabajadores resaltan que sus acciones colectivas se orientan a la defensa del derecho a la salud de la población. Afirman que sus demandas no son únicamente sectoriales sino también por mejorar la salud pública de todos los neuquinos. Esto se ve en los siguientes fragmentos de entrevistas y discursos públicos:

Como trabajadores de la salud pública venimos reclamando permanentemente al gobierno que se ocupe del sistema público de salud, y manifestando la necesidad de un amplio debate sobre una reforma sanitaria que tenga como eje el fortalecimiento de la salud pública, gratuita, universal y de calidad para todos, para toda la población, así como también la necesaria jerarquización del recurso humano, pilar esencial de la misma, a través de salarios dignos y la erradicación de toda forma de precarización laboral. (Marcelo, médico clínico, miembro de SiProSaPuNe)

La lucha permanente de los trabajadores de salud neuquinos es un compromiso real con la defensa del derecho a la salud de todos los ciudadanos. (Comunicado de prensa de la SiProSaPuNe, año 2007).

Que "la comunidad" aparezca en la disputa descansa en un proceso que es a la vez afectivo y performativo. Los enunciados performativos son actos del habla en el sentido que la acción se realiza se completa en el propio acto de enunciación (por ejemplo, afirmar "yo juro"). La apelación a la comunidad es un acto discursivo performativo laxo en el sentido que pretende hacer suceder aquello que nombra, cuestión que tiene lugar por los sentidos compartidos con los interlocutores. Los trabajadores del HPN se refieren a un colectivo abstracto que ellos mismos intentan construir. 
Si el carácter performativo de la apelación a la comunidad es exitoso, no se debe a que se base únicamente en un buen cálculo estratégico, sino a que activa narrativas y sensibilidades compartidas con el auditorio. La relación con la sociedad neuquina no debe verse únicamente como una estrategia de alianza política basada en un cálculo racional de los trabajadores del HPN, puesto que también se relaciona con cómo los trabajadores se piensan y sienten a sí mismos. Como se ve en el siguiente fragmento, los trabajadores se sienten parte de dicha comunidad: "Nosotros como trabajadores tenemos que tratar bien a la comunidad, porque vos sos parte de la comunidad.” (José, auxiliar técnico de electro medicina, miembro de ATE)

Asimismo, esta relación se expresa en determinados momentos y con determinadas palabras. No aparece de forma descontextualizada sino que es nombrada junto con afirmaciones como "defensa del sistema de salud”, "derechos sociales", "contra el vaciamiento que buscan los funcionarios”. El hecho de presentar sus demandas gremiales como demandas más amplias que abarcan al conjunto de "la comunidad" de neuquinos tiene eficacia política porque activa mediaciones compartidas con dicha comunidad; es decir, la responsabilidad de luchar contra el avasallamiento del derecho a salud pública es creíble tanto para los trabajadores como para las organizaciones que los acompañan y apoyan sus huelgas. Los trabajadores se sirven de un repertorio de categorías que expresan ideas y valores compartidos con el auditorio, pues no hay eficacia política posible si el público no adhiere a los presupuestos que sostienen los discursos de los oradores en los actos políticos (Soprano, 2005).

A partir de este proceso los trabajadores del HPN delimitan distintos grupos y espacios del Estado provincial, creando identificaciones sociales a partir de las cuales los actores se definen a sí mismos -trabajadores y comunidad- y definen a los otros -funcionarios de gobierno-. Las tensiones y disputas presentes la experiencia de los trabajadores permiten pensar al hospital como el resultado de un heterogéneo entramado de en disputa, que tienen sentido en contextos localmente situados. Deben considerarse las disputas entre distintos grupos de personas, así como su impacto de las mismas en los procesos de organización y sociabilidad de "los trabajadores".

\section{La imbricación de lo político y la política.}

Los aspectos analizados muestran que el hospital, como otros espacios de trabajo estatales, no es únicamente un espacio laboral sino también un lugar donde se aplica y gestiona la política pública. A diferencia de los ámbitos de trabajo privados, aquí la intervención de los trabajadores en sus espacios cotidianos laborales implica necesariamente un vínculo directo con la implementación de las políticas estatales -en este caso, las políticas sanitarias del Estado Provincial de Neuquén-. El conflicto político del que participan los trabajadores del HPN se presenta en dos planos: por un lado, los trabajadores tienen demandas estrictamente gremiales vinculadas a las condiciones laborales del sector, y por otro lado, ponen en discusión aspectos más amplios de la gestión política de los servicios estatales.

Como hemos visto, es usual que los "planes de lucha” de los trabajadores del HPN estén articulados en torno a la demanda general de "recomposición salarial” y mejoramiento de las condiciones laborales dentro del trabajo hospitalario: demanda de recomposición de la carrera sanitaria y re-categorización para los trabajadores de salud pública, denuncia de los procesos de precarización laboral que sufren algunos trabajadores (por el régimen de residencia médica, las modalidades de contrato mensuales, la inexistencia de francos-calendario, la insalubridad de las tareas). Pero además, suele incorporarse la denuncia de vaciamiento de ciertas políticas públicas, visible en la falta de insumos y aparatología para el sistema público de salud, o las falencias de infraestructura. Vemos que si bien algunas de estas demandas reflejan intereses corporativos propios de los trabajadores (como las condiciones de venta de la utilización de la fuerza de trabajo) y otras dejan ver el conflicto presente en el desenvolvimiento de las políticas públicas.

Si bien hay otros sectores de la población neuquina que articulan sus demandas sectoriales a disputas más 
generales por la política pública, lo que es distintivo en este caso es que los trabajadores del HPN demandan una respuesta al Estado provincial del que a su vez forman parte. A diferencia de los procesos de movilización social y protesta de otros sectores de la población neuquina, aquí no se trata de un vínculo simple entre la sociedad civil y el sistema político, pues las propias personas que se movilizan forman parte del entramado estatal. Consecuentemente, el desacuerdo con las políticas vinculantes no sólo se realiza desde fuera del sistema político sino también desde su interior.

Lefort (1992), afirma que la política tiene una doble inscripción: 1) Lo político es el momento de ruptura y renovación del orden social, donde la contingencia se hace presente a través de la visibilidad de las opciones posibles que pueden formar el orden; 2) La política es el lugar donde se ha normalizado lo político y se recrean los intercambios institucionalizados del conflicto; el espacio donde se oculta la contingencia radical del orden y se trata de domesticar las diferencias. Es decir, es el espacio donde se circunscribe la actividad política, la competencia por el poder y las decisiones políticamente vinculadas tomadas por los gobernantes $\stackrel{9}{\text {. }}$ En el análisis del conflicto sindical de los trabajadores del HPN encontramos estas dos dimensiones de la política articuladas: Por un lado, la participación política de los trabajadores del HPN podría ser descripta como la irrupción de lo político en tanto representa la visibilidad de los antagonismos sociales y la aparición de un discurso público sobre la salud dentro del escenario institucionalizado de la política. Sin embargo, la participación política de estos trabajadores también se puede describir como la presencia de un conflicto cotidiano en el escenario institucionalizado de la política, en tanto se trata de trabajadores pertenecientes al propio Estado provincial. La presencia del antagonismo en el HPN no sólo aparece cuando se suspende el trabajo para dar comienzo a una acción de protesta, sino también en el trabajo cotidiano. Con sus disputas, estos trabajadores no permiten que se domestiquen las diferencias en los intercambios institucionalizados del conflicto del Estado provincial; es decir, no permiten que la política domine la negatividad de lo político.

Por ende, para analizar la participación política de los trabajadores del HPN no se pueden observar los momentos de conflicto como un fenómeno diferente de las acciones cotidianas institucionalizadas del trabajo hospitalario. Si las huelgas son un momento productivo para analizar cómo se desenvuelve el conflicto social del que participan los trabajadores del HPN no se debe a que sea el único momento donde aparezca el conflicto. Las políticas públicas (tanto laborales como relativas a la gestión de la salud pública) no se disputan únicamente cuando se paraliza el trabajo, sino también en las labores cotidianas del hospital. Al analizar las prácticas políticas de los trabajadores del HPN vemos que la participación en redes de politicidad no es algo extra-cotidiano, sino que forma parte del proceso mismo en que se desenvuelve el trabajo.

Por ello considero necesario no analizar únicamente las acciones colectivas disruptivas en el espacio público y los momentos de huelga, sino también el entramado particular de disputa cotidiana que establecen los trabajadores en relación al Estado provincial. Por ello, consideramos necesario conceptualizar el Estado como un campo de fuerzas que es el resultado de múltiples presiones (Manzano, 2008). Los ámbitos estatales de trabajo no son instituciones autónomas con funciones específicas dentro de la vida social, y la política pública no se desarrolla tampoco como un proceso lineal que inicia con el diseño por parte de los funcionarios y termina por la aplicación en manos de los trabajadores. Los ámbitos estatales de trabajo son más bien un campo de fuerzas donde los distintos balances de poder van definiendo históricamente las formas legítimas de actuación estatal. Las prácticas políticas de los trabajadores del HPN muestran que, tal como lo han señalado Frederic y Soprano (2008), la disputa por control de los recursos humanos, materiales y simbólicos conlleva a que el diseño y ejecución de políticas públicas sean un resultado negociado entre actores sociales con desigual poder pero igualmente implicados. Es conveniente entonces analizar los ámbitos estatales de trabajo de forma contextual y temporal; es decir, como un fenómeno cambiante.

En suma, la experiencia gremial de los trabajadores del HPN nos muestra particularidades del sindicalismo dentro del empleo público que deben tenerse en cuenta dentro de los análisis de ciencias sociales. Por un 
lado, aquí se registra una articulación entre los aspectos estrictamente gremiales relativos a la búsqueda de mejorías en las condiciones de trabajo en el sector y las disputas más generales sobre la gestión de la política pública. Por otro lado, en estos casos el conflicto con las autoridades gubernamentales no aparece únicamente en los momentos de huelga o paralización del proceso de trabajo, sino que se registra en el trabajo cotidiano dentro de las instituciones estatales.

\section{Reflexiones finales. Claves para pensar el conflicto sindical de trabajadores de salud pública.}

Más allá de la reflexión específica sobre este hospital, el estudio de las tramas de politicidad allí presentes son útiles para aportar de manera más general al análisis del conflicto sindical de trabajadores de salud pública:

En primer lugar, hemos visto que el sistema de salud pública posee regulaciones legales especiales tanto para el desarrollo de los servicios como para los derechos sindicales de los trabajadores. La obligación de mantener servicios mínimos no es sólo un dato contextual sino que es una cuestión incorporada a las formas de participación políticas de los trabajadores, a sus articulaciones con otros actores, a sus sentimientos, etc. Son cuestiones que impactan en sus redes de sociabilidad y politicidad. Por ello es conveniente estudiar los procesos de movilización de los trabajadores de salud pública en relación a las reglas específicas que regulan su trabajo y a cómo son usadas por los trabajadores.

En segundo lugar, vimos que en el conflicto sindical de trabajadores de salud pública se da una articulación entre las demandas sindicales sectoriales y la disputa más general por la gestión de las políticas públicas, cuestión que en escasas situaciones es visible en los conflictos sindicales de trabajadores formales del sector privado. Los reclamos sindicales de los trabajadores del HPN se encuentran divididos por la particularidad que encarnan y la significación más universal de la que son portadores. Los conflictos sindicales en estos casos tienen una relación directa con la disputa por los bienes comunes, cuestión que favorece que se ponga en discusión las formas estatales de gestión de lo público. Por eso mismo, la presencia de la comunidad a la que se orientan los servicios estatales tiene una importancia decisiva en el desenvolvimiento del conflicto. Las tramas políticas de los trabajadores del HPN no se circunscriben al interior del hospital, sino que se extiende a organizaciones externas. Con "la comunidad" no se establece una relación de alianza estratégica únicamente, sino que se ponen en juego aspectos de la sensibilidad y afectividad compartidos por los trabajadores en relación a los derechos sociales que están en juego.

En tercer lugar, hemos visto que las demandas de los trabajadores no pueden evaluarse como un fin que dependa únicamente de su decisión estratégica individual o sectorial, sino que debe ser analizada en relación a los múltiples conflictos cotidianos que surgen en el entramado hospitalario. Es conveniente analizar la disputa que establecen los trabajadores en relación al Estado, conceptualizándolo como un campo de fuerzas que es el resultado de múltiples presiones. La participación sindical de los trabajadores estatales implica una articulación de dos dimensiones de la política que en otros casos aparecen separadas: por un lado, puede ser descripta como la irrupción de lo político en tanto representa la visibilidad de los antagonismos sociales y la aparición de un discurso público dentro del escenario institucionalizado de la política; pero por el otro lado, también se puede describir como la presencia de un conflicto cotidiano en el escenario institucionalizado de la política, en tanto se trata de trabajadores pertenecientes a las propias estructuras estatales. Con sus disputas, estos trabajadores no permiten que se domestiquen las diferencias en los intercambios institucionalizados del conflicto del Estado; es decir, no permiten que la política domine la negatividad de lo político.

El análisis de la experiencia sindical de los trabajadores del HPN permite realizar aportes a los estudios sindicales desarrollados en la post-convertibilidad en tanto se analizan empíricamente dos aspectos que han quedado su representados en estos estudios: por un lado, el análisis de las particularidades que adquiere el 
conflicto sindical de trabajadores estatales (en relación a los sindicatos del sector privado); y por el otro, registrar empíricamente los procesos de movilización y organización de trabajadores del interior del país. El análisis de estos elementos requiere de una perspectiva de análisis del conflicto sindical que no se centre únicamente en el estudio de los momentos de huelga, sino que atenga a la dinámica cotidiana de relaciones en las que se inserta la participación sindical de los trabajadores. El enfoque analítico aquí presentado supone un abordaje relacional y situado que atienda a la configuración de la acción colectiva y los momentos de huelga en el entramado de relaciones del que forma parte.

\section{Notas}

1 La fuerza que adquirieron las negociaciones tripartitas en este proceso no debe ocultar sin embargo que existen grandes fracciones sociales que quedan por fuera del derecho colectivo (Marticorena, 2011), como los trabajadores informales y no registrados. Por ello, diversos investigadores resaltaron la necesidad de atender a las acciones colectivas de trabajadores informales y/o precarios (Busso, 2007) y la emergencia de resistencias más individualizadas y localizadas en los espacios de trabajo (Montes Cató, 2006).

$\underline{2}$ Estudios interesantes de estos procesos han sido desarrollados por investigadores locales (Ver Aiziczon, 2006, 2009; Bonifacio, 2011; Burton, 2012; Burton \& Rosales, 2010; Lizarraga, 2010; Petruccelli, 2005).

$\underline{3}$ Algunos de los aspectos que se mencionan a continuación han sido analizados en mayor profundidad en Beliera (2015, 2016).

4 Diversas organizaciones tienen locales sindicales en este hospital: la Asociación de Trabajadores del Estado (ATE), la Unión del Personal Civil de la Nación Sector Salud (UPCN), y el Sindicato de Profesionales de la Salud de la Provincia del Neuquén (SiProSaPuNe). Además, hay fuerte presencia del Sindicato de Enfermeros de Neuquén (SEN), cuya mayor cantidad de afiliados corresponde a este hospital.

$\underline{5}$ En la elaboración de bases sobre conflictividad laboral han sido centrales las tareas el equipo de investigación sobre conflicto laboral del Ministerio de Trabajo, Empleo y Seguridad Social de la Nación. Ver (Palomino \& Trajtemberg, 2007).

$\underline{6}$ Algunas dificultades son metodológicas (debido a la falta de fuentes o a la ausencia de series históricas para comparar) y otras están vinculadas a las características del modelo sindical argentino y/o del mercado de trabajo (pues la fuerte segmentación del mercado laboral hace que las mejorías en las condiciones laborales establecidas en los convenios colectivos de trabajo solo afecten a los trabajadores formales, quedando por fuera el gran número de trabajadores informales o precarios que componen la economía local). Ver Atzeni \& Ghigliani (2008), Ghigliani (2014).

7De esta forma, la Argentina se aparta de la recomendación efectuada por la Comisión de expertos de la OIT en 2003, según la cual la determinación de los servicios mínimos no debería corresponder al Ministerio de Trabajo sino a un órgano independiente (Centro de Estudios para el Desarrollo Argentino, 2006) .

$\underline{8}$ Esta huelga ha sido analizada en Beliera (2013).

$\underline{9}$ Un análisis sobre las diversas formas en que se ha teorizado sobre esta doble inscripción de lo político (como momento de la institución y de lo instituido, como acto abismal y como subsistema, como lo político y lo social, etc.) ha sido teorizada por Arditi (2005). 


\section{Fuentes primarias}

-Ley 25.877, sancionada en el año 2004

-Revista sindical Escepticemia, folletín elaborado por la Asociación de Profesionales del HPN. Periodo 1999-2007

-Archivo periodístico del Diario Rio Negro de la provincia de Neuquén desde setiembre del año 2004 hasta diciembre del año 2005

\section{Referencias bibliográficas}

Aiziczon, F. (2006). Protesta social y cultura política. Aportes para pensar los años '90 en Neuquén. Ponencia presentada en las II Jornadas Patagónicas de Historia., Fiske Menuko, Rio Negro. Recuperado a partir de http://goo.gl/FYaDJl

Aiziczon, F. (2009). Zanon, una experiencia de lucha obrera. Buenos Aires: Herramienta \& El Fracaso.

Anigstein, C. (2012). La revitalización de la negociación colectiva en la argentina y sus controversias. Ponencia presentada en VII Jornadas de sociología UNGS, Universidad de General Sarmiento.

Antón, G., Cresto, J., Rebón, J., \& Salgado, R. (2010). Una década en disputa. Apuntes sobre las luchas sociales en la Argentina. OSAL, XI (28), 94-115.

Arditi, B. (2005). El devenir-otro de la política. Un archipielago post-liberal. En ¿Democracia post-liberal? El espacio político de las asociaciones. Bacelona: Anthropos.

Arias, C. C., \& Haidar, V. (2008). Resistir en nombre de la salud. Un análisis de la experiencia del Cuerpo de Delegados de los subterráneos de Buenos Aires. Trabajo y Sociedad. Recuperado a partir de http://www.redalyc.org/articulo.oa?id=387334683004

Atzeni, M., \& Ghigliani, P. (2008). Nature and limits of trade unions’ mobilisations in contemporary Argentina. Labour Conflicts in Contemporary Argentina. Labour Again Publications. Recuperado a partir de https://www.researchgate.net/profile/Pablo Ghigliani/publication/254000896 Nature and limits of trade u nions' mobilisations in contemporary Argentina1/links/5630bcfa08ae336c42eb55af.pdf

Battistini, O. (2010). Tiempos de cambio para viejas estructuras. El modelo sindical en crisis. Recuperado a partir de http://goo.gl/E5Xnir

Beliera, A. (2013). «En defensa de la salud pública». Notas sobre las acciones colectivas de los/as trabajadores/as del Hospital Castro Rendón frente a las reformas neoliberales. Trabajo y sociedad, 20, 355373.

Beliera, A. (2015). Entramado de politicidad en un ámbito de trabajo: la experiencia de los trabajadores del Hospital Provincial Neuquén (2005-2013) (Tesis de Maestría). Universidad Nacional de La Plata, La Plata.

Beliera, A. (2016). Pensar al Estado como ámbito de trabajo. Reflexiones a partir de la experiencia de trabajadores de salud pública de Neuquén (2005-2013). Estudios Sociales del Estado, 2(3), 180-211.

Bonifacio, J. L. (2011). Protesta y organización: los trabajadores desocupados en la provincia de Neuquén. Buenos Aires: El Colectivo.

Burton, J. (2012). Lo unico que han conseguido es despertar a la bestia. Una modalidad de respuesta al conflcito docente en el Neuquén de la confianza. (Tesis de grado de la Licenciatrua de Sociología). Universidad Nacional del Comahue, Fiske Menuco, Rio Negro. 
Burton, J., \& Rosales, L. (2010). Tensiones y debates en torno al derecho a la educación: estrategias gubernamentales para desacreditar el conflicto docente neuquino, marzo - mayo 2010. Presentacion en las VI Jornadas de Sociologia UNLP, La Plata, Argentina.

Busso, M. (2007). Trabajadores informales en Argentina: ¿de la construcción de identidades colectivas a la constitución de organizaciones? (Tesis doctoral en cotutela). UBA -Université de Provence.

Cambiasso, M. (2013). Estrategias político-sindicales, experiencias de lucha y tradición de organización en la Comisión Interna de Kraft-Terrabusi (Tesis de Maestría). Universidad Nacional de Buenos Aires., Buenos Aires.

Campos, L. (2012). La negociación colectiva en la posconvertibilidad: recuperación histórica y acumulación de tensiones. Revista Digital de EconomÍa Politica.

Campos, L., \& Campos, J. (2011). El conflicto laboral y la negociación colectiva durante 2010 en Argentina. En Jornadas de Economía Crítica. Córdoba.

Centro de Estudios para el Desarrollo Argentino. (2006). Informe laboral numero 10 (El trabajo en Argentina, condiciones y perspectiva.). Buenos Aires.

Compañez, M., Ventrici, P., \& Voscos, F. (2012). Metrodelegados. Subte: de la privatización al traspaso. Buenos Aires: Desde el subte.

Coscia, V. (2011). «Resurgimiento» de sindicatos tradicionales y nuevas formaciones sindicales en Argentina. En XXVIII Congreso Internacional de la Asociacion Latinoamericana de Sociología (ALAS).

Recife, Brasil.

Criado, E. M. (2008). El concepto de campo como herramienta metodológica. Revista Española de Investigaciones Sociológicas (REIS), 123, 11-33.

Del Bono, A. (2013). Negociación colectiva, tercerización y modalidades de contratación atípicas. En Negociación colectiva y representación sindical. Asociación Argentina de Especialistas en Estudios del Trabajo (ASET) y Friedrich Ebert Stiftung.

Diana Menéndez, N. (2007). La representación sindical en el Estado: los casos de la Asociación de Trabajadores del Estado (ATE) y la Unión del Personal Civil de la Nación (UPCN). Presentado en 7o Congreso Nacional de Estudios del Trabajo, Buenos Aires, Buenos Aires. Recuperado a partir de http://www. aset. org. ar/congresos/7/12001.

Duhalde, S. (2011). Las potencialidades políticas de lo gremial y sus límites. Un caso de sindicalismo clasista: Ate-Garrahan 2003-2010. Sociohistórica, 0(30). Recuperado a partir de http://www.sociohistorica.fahce.unlp.edu.ar/article/view/SHn30a06

Elias, N. (1999). Sociología fundamental. Barcelona: Gedisa.

Etchemendy, S. (2011). El Dialogo Social y las Relaciones Laborales en Argentina 2003-2010. Estado, sindicatos y empresarios en perspectiva comparada (Vols. 1-1a). Buenos Aires: Oficina Regional para América Latina y el Caribe, Programa Regional para la Promoción del Diálogo y la Cohesión Social en América Latina.

Etchemendy, S., \& Collier, R. B. (2008). Golpeados pero de pie. Resurgimiento sindical y neocorporativismo segmentado en Argentina (2003-2007) -. POSTData: Revista de Reflexión y Análisis Político. Recuperado a partir de http://goo.gl/vCKDPa

Fernández Álvarez. (2007). De la recuperación como acción a la recuperación como proceso: prácticas de 
movilización social y acciones estatales en torno a las recuperaciones de fábricas. Cuadernos de antropología social, (25), 89-110.

Frederic, S., \& Soprano, G. (2008). Construcción de escalas de análisis en el estudio de la política en sociedades nacionales. En Política y variaciones de escalas en el análisis de la Argentina. Buenos Aires: Universidad Nacional de General Sarmiento.

Ghigliani, P. (2014). Acerca de los estudios cuantitativos sobre conflictos laborales en Argentina (19732009): reflexiones sobre sus premisas teórico-metodológicas. Conflicto Social, 2(2), 75-97.

Gómez, M. (2006). Crisis y recomposición de la respuesta estatal a la acción colectiva desafiante en la Argentina (1989-2004). Revista argentina de sociología, (6), 88-128.

González, S. S., \& Haidar, J. (2009). Los debates acerca de la «revitalización sindical» y su aplicación en el análisis sectorial en Argentina. Revista Latinoamericana de Estudios del Trabajo, ea Epoca(22), 5-31.

Laclau, E. (2004). Discurso. Topos\&Tropos, 1(7).

Laclau, E. (2005). La Razón Populista. Buenos Aires: Fondo de Cultura Económica.

Laclau, E., \& Mouffe, C. (1985). Hegemonía y estrategia socialista. Buenos Aires: Fondo de Cultura Económica.

Lefort, C. (1992). La representación no agota a la democracia. En M. Dos Santos, ¿¿ué queda de la representación política? Buenos Aires: Nueva Sociedad.

Lenguita, P. (2011). Revitalización desde las bases del sindicalismo argentino. Revista Nueva Sociedad, 232.

Lizarraga, F. (2010). Sobisch, la neuquinidad y la construcción del enemigo absoluto. En O. Favaro \& G. Iuorno, El arcón de la historia reciente en la Norpatagonia argentina (pp. 23-55). Buenos Aires: Biblos.

Manzano, V. (2008). Del desocupado como actor colectivo a la trama política de la desocupación: antropología de campos de fuerzas sociales. En M. Cravino, Acción colectiva y movimientos sociales en el Área Metropolitana de Buenos Aires. General Sarmiento: UNGS.

Marticorena, C. (2011). Contrapuntos de la negociación colectiva en la industria manufacturera durante el período pos convertibilidad. Ponencia presentada en X Congreso Nacional de Estudios del Trabajo, Buenos Aires. Recuperado a partir de http://goo.gl/NswIEv

Marticorena, C. (2013a). Los contenidos de la negociación colectiva durante la postconvertibilidad. Características e interrogantes. En Negociación colectiva y representación sindical. Asociación Argentina de Especialistas en Estudios del Trabajo (ASET) y Friedrich Ebert Stiftung.

Marticorena, C. (2013b). Relaciones laborales y estructura de la negociación colectiva. Avances en torno a su problematización. En 11 Congreso Nacional de Estudios del Trabajo. Buenos Aires.

Montes Cató, J. (2006). Dominación y resistencia en los espacios de trabajo. Estudio sobre las relaciones de trabajo en empresas de telecomunicaciones (Tesis Doctoral). Facultad de Ciencias Sociales. Universidad de Buenos Aires., Buenos Aires.

Palomino, H., \& Trajtemberg, D. (2007). Un nuevo indicador del Ministerio de Trabajo, Empleo y Seguridad Social. Los conflictos laborales en la Argentina 2006-2007 (Trabajo, ocupación y empleo. Los retos laborales en un proceso de crecimiento sostenido).

Petruccelli, A. (2005). Cap 1: El escenario y sus actores. En Docentes y piqueteros. De la huelga de ATEN a la pueblada de Cutral-Có. Buenos Aires: El Fracaso/ El cielo por asalto. 
Santella, A. (2011). La representación sindical en los establecimientos. Antecedentes, problemas y discusiones. El modelo sindical en debate, ASET, 31-51.

Scolnik, F. (2009). El movimiento obrero argentino entre dos crisis: las organizaciones de base antiburocráticas en el área metropolitana de Buenos Aires durante el período 2003-2007. Conflicto Social, 2(2). Recuperado a partir de http://goo.gl/ntaqPv

Soprano, G. (2005). La producción de actores e identidades políticas en el peronismo durante una campaña electoral. En S. Frederic \& G. Soprano, Cultura y política en etnografías sobre la Argentina. Bernal: Editorial de la Universidad de Quilmes.

Trajtemberg, D. (2013). Estructura y determinantes de la negociación colectiva en Argentina. En Negociación colectiva y representación sindical. Asociación Argentina de Especialistas en Estudios del Trabajo (ASET) y Friedrich Ebert Stiftung.

Varela, P. (2016). La resistencia de los trabajadores precarizados en el sindicalismo de base en Argentina: apuntes sobre las experiencias de Subte, Kraft y Madygraf (ex Donnelley). Revista Pilquen. Sección Ciencias Sociales, 19(3), 66-78. 